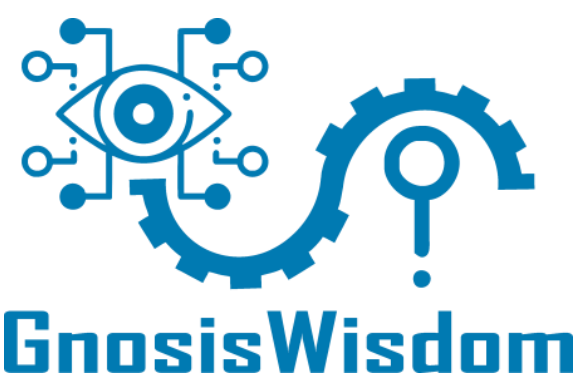

Artículo original

Volumen 1, Número 2, Mayo - Agosto 2021

\title{
COMUNICACIÓN NO VERBAL SEGÚN LA PERSPECTIVA TEÓRICA DE VIGOTSKY EN LA UNIVERSIDAD NACIONAL DE HUANCAVELICA: ESTUDIO DE CASO
}

\section{NON-VERBAL COMMUNICATION ACCORDING TO VIGOTSKY'S THEORETICAL PERSPECTIVE AT THE NATIONAL UNIVERSITY OF HUANCAVELICA: A CASE STUDY}

\section{Autores}

Sánchez Gonzales, Antonia del Rosario ORCID

Instituto de Educación Pedagógico Público Teodoro Peñaloza

Arias Sánchez, Raúl Eleazar ORCID

Universidad Nacional de Huancavelica

\section{Resumen}

La presente investigación tuvo por objetivo realizar una aproximación a las formas de manifestación de comunicación no verbal desde la perspectiva teórica de Vygotsky en los estudiantes, docentes y personal administrativo de la Facultad de Ciencias Empresariales de la Universidad Nacional de Huancavelica mediante un enfoque cualitativo y casuístico.

Palabras clave: Comunicación, teoría, enfoque

\begin{abstract}
This paper was to make an approach to the forms of manifestation of non-verbal communication since Vygotsky's theoretical perspective in students, teachers and administrative staff at Faculty of Business Sciences at Universidad Nacional de Huancavelica through a qualitative and casuistic approach.
\end{abstract}

Keywords: Communication, theory, approach

\section{Introducción}

El psicólogo ruso de origen judío Lev Semiónovich Vygotsky $^{1}$ argumentaba que nuestros pensamientos y lenguaje formaban parte de nuestras funciones mentales superiores y que eran de vital importancia para entender y comprender todo acto de aprendizaje

${ }^{1}$ Fundador de la psicología histórico-cultural y precursor de la neuropsicología soviética. 


\section{GnasisWisdam}

en los diversos grupos humanos asentados en el globo; sobre la última función, Álvarez (2010, citado de Mehler y Dupoux, 1990) manifestó que, “...el lenguaje no es la única habilidad cognitiva que tenemos. Es una más entre todo un entramado de capacidades que abarcan la percepción, la memoria, la atención, el razonamiento, el pensamiento, la capacidad de cálculo y el resto de lo que podríamos llamar conductas o habilidades inteligentes. Dicho de otra forma, la "inteligencia", para la mayor parte de científicos cognitivos, no es una aptitud única y transparente, sino que la cognición implica numerosos sistemas especializados y relativamente autónomos. Sin embargo, no es menos cierto que estas capacidades autónomas interactúan continuamente entre sí. Por ello, investigar la dependencia/independencia entre el lenguaje y el resto de la vida mental es un objetivo prioritario para todos aquellos interesados en la Lingüística y Psicología del Lenguaje, en particular, en la cognición humana, en general, tal y como lo fue para Lev Vigotsky." (pp. 16-17)

Como vemos, esta perspectiva teórica sobre la habilidad del lenguaje ha sido enfrentada y demuestra claramente que esta capacidad está constituida por un mosaico de procesos mentales que hacen posible la creación de enunciados lingüísticos y no lingüísticos los cuales poseen un sustento de investigación cualitativa fundamentada en la interacción social y contexto sociocultural a través de la observación. Sin embargo, los productos resultantes de esta capacidad son variados y se encaminan a convertirse en un tema de investigación y diálogo interdisciplinario. Para Chaves (2001) aportes teóricos como la perspectiva de capacidades y enunciados similares como los de esta pesquisa representarían:

“...propuestas pertinentes para repensar la educación y la práctica pedagógica. Estos postulados coinciden en la importancia de respetar al ser humano en su diversidad cultural $y$ de ofrecer actividades significativas para promover el desarrollo individual y colectivo con el propósito de formar personas críticas y creativas que propicien las transformaciones que requiere nuestra sociedad." (p. 63)

En este sentido, conocedores de nuestra diversidad, hemos visto por conveniente estudiar una actitud de ésta, la cual está presente dentro de los miembros de nuestra comunidad universitaria, la comunicación no verbal. Esto, siguiendo las pautas de Castorina y Dubrovsky (2004) quienes recomendaron desarrollar la perspectiva vigotskyana de capacidades con un enfoque social e interrelacionado con sujetos particulares de grupos organizados.

Sobre lo planteado, es relevante indicar que, para su comprensión y análisis es necesario delimitar una definición y posterior escenario de estudio. Así, indicamos que la comunicación es una cualidad inherente en los hombres de todas las condiciones y sociedad. Ésta le ha permitido desde siempre relacionarse con su entorno e interactuar son sus semejantes (Freixas, 2017).

Hoy en día, la especialización del conocimiento nos permite ver de forma más objetiva este proceso y las diversas manifestaciones que tiene en nuestras vidas y cotidianidad. Un claro ejemplo de ello es la 


\section{GnasisWisdam}

denominada Comunicación No Verbal (CNV) la cual es motivo de nuestras interrogantes. La comunicación no verbal a nuestro entender podría definirse como (Cabana, 2008, citado por Rodríguez y Hernández, 2010):

“...una forma de interacción silenciosa, espontánea, sincera y sin rodeos. Ilustra la verdad de las palabras pronunciadas al ser todos nuestros gestos un reflejo instintivo de nuestras reacciones que componen nuestra actitud mediante el envío de mensajes corporales continuos. De esta manera, nuestra envoltura carnal desvela con transparencia nuestras verdaderas pulsiones, emociones y sentimientos. Resulta que varios de nuestros gestos constituyen una forma de declaración silenciosa que tiene por objeto dar a conocer nuestras verdaderas intenciones a través de nuestras actitudes" (p. 6)

Corrales (2011) indicó que CNV, surgió en la edad más temprana de la humanidad mucho antes de que nuestro lenguaje y que sus diversas formas evolucionaron generación tras generación. Considerando esta premisa, recurrimos a la postura darwiniana ${ }^{2}$, la cual indica al respecto que se puede decir que la forma de comunicarnos de manera no verbal con algunas emociones como el enojo, el miedo o la alegría, son innatas y heredadas por nuestros ancestros gracias a la evolución, y que, las compartimos con los primates y algunos mamíferos superiores, sin embargo, su reconocimiento y presencia son patrones de cultura aprendidos y transmitidos de los más viejos hacia los más jóvenes directa e indirectamente. En este sentido, nuestra investigación se orientó a registrar y describir los tipos frecuentes de comunicación no verbal en diferentes estados de ánimo como el mal humor, la alegría y la tristeza de un grupo de estudiantes, docentes y personal administrativo de las escuelas profesionales de Economía, Administración y Contabilidad de la Facultad de Ciencias Empresariales de la Universidad Nacional de Huancavelica, quienes nos brindaron su consentimiento y apoyo para llevar a cabo esta investigación.

\section{Material y método}

Como método general de investigación fue empleado el método científico y como específico el método etnográfico el cual es entendido como "el estudio directo de personas y grupos durante un cierto periodo, utilizando la observación participante o las entrevistas para conocer su comportamiento social, registrando una imagen realista y fiel del grupo estudiado; el trabajo de campo resulta ser una herramienta imprescindible" (Giddens, 2007, p.27).

De igual forma, esta metodología cualitativa vio por conveniente recoger información a través de la observación participante y entrevistas, las cuales ayudaron a acercamiento a nuestra muestra, tratando de aproximarnos a la naturaleza de su conducta (estudio de casos). Asimismo, nuestra pesquisa comenzó con el diseño de una ficha de entrevista online a través de la plataforma Google Forms cuyo número de preguntas fue de 3; de igual forma, comprendió información sociodemográfica como edad, sexo, ciclo, carrera

\footnotetext{
${ }^{2}$ Es importante destacar, que toda conducta cultural tiene una base honda en nuestra biología. (Darwin, 1872)
} 


\section{Gnasis Wisdom}

profesional, tiempo de trabajo, y oficina correspondiente con la finalidad de realizar una prueba piloto enfatizando su carácter confidencial y anónimo de la encuesta diseñada. Finalmente, nuestra entrevista se realizó a través de la plataforma de videoconferencia Google Meet. Debemos de señalar también que, tuvimos el consentimiento verbal de cada persona que accedió a ser entrevistada por nosotros para grabarlas y como parte de nuestra ética profesional les garantizamos la total confidencialidad con sus datos (en la gran mayoría de casos). Cada entrevista tuvo la duración aproximada de $15^{\prime}$ a $20^{\prime}$ minutos; éstas se convirtieron en conversaciones horizontales y asertivas, asimismo, cada experiencia relatada fue transcrita y tabulada de forma descriptiva para poder compararlas y llegar así a las conclusiones que se verterán en las próximas líneas.

\section{Resultados/discusión}

Debemos de señalar que la expresión gestual de las emociones es fundamental para todo tipo de interacción en nuestra sociedad y su forma de ser exteriorizada depende mucho de cómo la hemos aprendido mediante el proceso de endoculturación. Por ejemplo, ningún trabajador entrevistado se atreverá a pedirle un aumento y/o préstamo de dinero a su jefe si lo observa arrugando un papel con fuerza, conversando por el celular, con los ojos "chinos" o con el ceño poco amigable. Indicaciones claras que algo no anda bien, y que es mejor intervenir o conversar en otro momento. $\mathrm{Al}$ respecto hemos formulado algunas preguntas que

${ }^{3}$ Entrevista anónima realizada el día 07 de septiembre de 2020, horas 4:07 pm.

${ }^{4}$ Entrevista anónima realizada el día 08 de septiembre de 2020, horas 7:32 pm. fueron contestadas por nuestra muestra y la cuales las compartiremos a continuación:

a. Ante la pregunta: ¿cómo sabes que tu jefelprofesor está de mal humor?, obtuvimos respuestas como:

- “...me doy cuenta cuando se achina (cierra sus ojos), ahí es mal momento para preguntarle algo...prefiero no ir porque si no se paga conmigo..."3

- "...el profe se molesta cuando estira sus manos en su mesa y se estira, eso pasa cuando no presentamos trabajo, parece que no pasa nada, pero cuando hace eso ya es fijo que vamos a dar examen (sorpresa) ..." 4

- “...cuando mi papá está almorzando y alguien dice algo que no le agrada, deja de comer y nos mira...a veces me da miedo...calladitos todos después de eso..."

- “...mis viejitos son la canela,...cuando se molestan se miran entre ellos, y fijo se enteraron algo que hice (risas)..."6

Sobre lo vertido, podemos decir que tanto nuestras manos como nuestros ojos presuponen características que podemos interpretar como buenas o malas según el contexto. 


\section{GnasisWisdam}

b. Ante la pregunta: ¿cómo sabes que tu jefe/profesor/familiar está alegre?, obtuvimos respuestas como:

- "...cuando entra silbando a la oficina quiere decir que está alegre...fijo fijo le salió algo (bueno), ya lo conozco un año, ya sé cómo es..."7

• “...bueno, el profe siempre está alegre, lo que sí es cuando está triste su voz es un poco menos, como que, si estuviese mal de la garganta algo así, pero por lo general siempre está alegre..." 8

- “...mi hermano está alegre cuando quiere cocinar papa frita con atún, cuando le pagan por algo, así es lo es...otras veces también pone música esto en inglés...así sabemos que está bien..."

- “...cuando le escucho por teléfono (a mi jefe) su voz cambia oye, de lo que me dice linda cómo estas, sólo me llama por mi nombre...ay no...algo seguro le habrá pasado pobrecito...luego ya cuando este le pasa...normal se siente..."10

En este escenario, podemos ver que nuestra voz puede generar en nuestro interlocutor un estado a interpretar ya sea de bienestar como no. Finalmente,

c. Ante la pregunta: ¿cómo sabes que tu jefe/profesor/familiar está triste?, obtuvimos respuestas como:

${ }^{7}$ Entrevista anónima realizada el día 06 de septiembre de 2020, horas 4:07 pm.

${ }^{8}$ Entrevista anónima realizada el día 09 de septiembre de 2020, horas 5:22 pm.

${ }^{9}$ Entrevista anónima realizada el día 05 de septiembre de 2020, horas 1:25 am.

${ }^{10}$ Entrevista anónima realizada el día 07 de septiembre de 2020, horas 6:49 pm.

${ }^{11}$ Entrevista anónima realizada el día 07 de septiembre de 2020, horas 9:32 am.
- “...noto que mi jefe está triste cuando cierra la puerta de su oficina y ni música pone, a veces entro a conversar ...creo que tienen problemas en su mujer...igual es bueno con su personal..."11

- “...(mi hermana) se agarra el pelo y da vueltas vueltas, así es su tristeza..." 12

- “...se tira en el sofá y ve películas repetidas, eso es que ya está mal, nos damos cuenta y mejor no decirle nada (a mi hermana) se molesta de todo en ese estado..."13

Ante esta última pregunta, podemos observar que la tristeza es mirada mediante la voz del interlocutor y en algunos escenarios es mejor dar y respetar el momento y espacio del mismo para aliviar su carga.

Esta pesquisa evidenció que la comunicación no verbal es aprendida, sin embargo, existen algunas situaciones a las que nuestra respuesta cultural es producto de nuestra base biológica como por ejemplo la tristeza asociada a un decaimiento de los ojos o que nuestra alegría resulta evidente por nuestra sonrisa. Entonces queda presente que aquello que Vygotsky llama como capacidades (y de forma específica la que evoca la comunicación no verbal) forman parte de nuestra cultura y que su desarrollo no solamente puede ser visto como una condición externa sino también interna. Nos parece que es el camino reflexivo al cual podemos apuntar.

\footnotetext{
${ }^{12}$ Entrevista anónima realizada el día 09 de septiembre de julio de 2020, horas 4:15 pm.

${ }^{13}$ Entrevista anónima realizada el día 10 de septiembre de julio de 2020, horas 3:22 pm.
} 


\section{GnasisWisdam}

Finalmente, podemos decir que, en base a estas tres preguntas, hemos podido reforzar más nuestro criterio de que existen gestos que exteriorizan un estado de ánimo como en las esferas familiar, estudiantil y laboral. Las expresiones que decimos con "nuestro cuerpo" sin duda indican algo que sucede dentro de nosotros, saber interpretarlos en sencillo siempre y cuando convivamos y compartamos con la persona un tiempo. Comprender estas "reglas" de la comunicación no verbal es vital para la interacción social en nuestro entorno. Conservarlas serán necesarias para continuar interactuando y conocernos mejor.

\section{Conclusiones}

- A nuestro criterio, la capacidad de comunicación no verbal desde la perspectiva teórica de Vygotsky es un resultado mixto entre condiciones externas como internas.

- Sobre lo compartido, podemos indicar que nuestros entrevistados reconocen ciertos gestos y actitudes que indican un estado de ánimo el cual se construyó en un tiempo determinado y mediante un proceso de socialización.

- La comunicación no verbal nos permite comunicar algo que quizá nos cuesta en palabras evidenciando emociones (procesos mentales) reflejadas en gestos (elementos físicos y perceptibles).

- Podemos decir también que, la convivencia con personas en entornos públicos como privados y/o familiares seguirá construyendo y creando más formas de comunicación no verbal entre los hombres.

\section{Referencias}

Álvarez González, C. J. (2010). La relación entre lenguaje y pensamiento de Vigotsky en el desarrollo de la psicolingüística moderna. RLA. Revista de lingüística teórica y aplicada, 48(2), 13-32.

Castorina, J. A., \& Dubrovsky, S. (2004). Psicología, cultura y educación: perspectivas desde la obra de Vigotsky. Noveduc Libros.

Corrales, E. (2011). El lenguaje no verbal: un proceso cognitivo superior indispensable para el ser humano. Revista Comunicación, vol. 20, núm. 1, enero-junio, 2011, pp. 46-51

Darwin, Ch. (1872). The Expression of Emotion in Man and Animals. Recuperado de: http://charles-darwin.classicliterature.co.uk/theexpression-of-emotion-in-man-andanimals/ebook-page-06.asp, el 27 de mayo de 2020

Freixas, C. P. (2017). Comunicación no verbal. Editorial Kairós.

Giddens, A. (2007). Sociología. Madrid, España: Alianza Editorial.

Rodríguez, I. y Hernández, M. (2010). Análisis de la comunicación no verbal de José Luis Rodríguez Zapatero. Revista Latina de Comunicación Social. (65). Universidad de La Laguna, p.p. 436 a 449.

Salas, A. L. C. (2001). Implicaciones educativas de la teoría sociocultural de Vigotsky. Revista educación, 25(2), 59-65. 